\title{
Automatic Detection of sunspot activities using advanced detection model
}

\author{
Manish T. I. ${ }^{1}$, D. Murugan ${ }^{2}$, Ganesh Kumar T. ${ }^{3}$ \\ ${ }^{1}$ Assistant Professor, Department of CSE, MET'S School of Engineering, India \\ ${ }^{2}$ Associate Professor, Department of CSE, Manonmaniam Sundaranar University, India \\ ${ }^{3}$ Research Scholar, Department of CSE, Manonmaniam Sundaranar University, India
}

\begin{abstract}
Sunspots are dark areas on the photosphere, from which sun emit light. Sunspots are regions located on active regions of the sun and have intense magnetic field [1]. Sunspots appear dark and bright areas in magnetograms representing opposite polarities. Despite several research and development in the field of pattern recognition with specific application like sunspots, the general problem of recognizing complex patterns remain difficult. A new technique was developed for automated detection of sunspots on full disk white light solar images obtained from SOHO/MDI and SDO/HMI instruments. Hurdles in the sun spot detection are the irregularities in the shape, contrast with the surrounding and uneven intensity make the sun spot detection difficult. In this paper we present a hybrid method to detect and extract features. The input is a sequence of MDI images and the output is categorization of solar events. We perform basic image processing techniques like normalization, noise removal and segmentation. Finally we compare the solar indices like wolf sunspot number with our method against with the synoptic maps and different reference observatory data source. The proposed method presented can lead to automatic monitoring and characterization of solar events and yield an optimum performance.
\end{abstract}

Keywords: Sunspots, Image enhancement, Edge optimization, Scale multiplication

\section{INTRODUCTION}

We require real time analysis and provide reliable predictions of forthcoming solar activities and their possible effects on Earth. Though the sun lies 149 million $\mathrm{km}$ from Earth, its constant activity assures an impact on our planet far beyond the obvious light and heat. The importance of understanding space weather is increasing because the way solar activity affects life on Earth and more on communications and power systems, both of which are vulnerable to space weather [1].Due to the increase in the size of data archives related to the solar, we need a automatic detection and analysis of solar data. The timely analysis of the solar data helps in reliable prediction of solar activity and its associated impact. While we go for the automatic detection scheme the accuracy of the prediction should be verified.

Sun spots contribute to the major solar activities, which has been studied and analyzed intensively nowadays. Sunspots are dark areas that grow and decay on the photosphere and appear dark because they are cooler than the surrounding photosphere, typically by about $1500 \mathrm{~K}$ which is cool compared to the rest of the photosphere layers. Sunspots develop and persist for periods ranging from hours to months, and are carried around the surface of the Sun by its rotation. A typical sunspot consists of a dark central region called the umbra and somewhat lighter surrounding region called the penumbra. The appearance and disappearance of sunspots is due to the varying magnetic field presence in the sun. This magnetic variation indicates the possibility of large amount of energy release from sun spots [2]. The sun spot may break away from original spot and form another sun spot. The International sunspot number is a quantity that measures the number of sunspots and groups of sunspots present on the surface of the sun. The sunspot activity is cyclical and reaches its maximum around every 9.5 to 11 years [3]. In this paper, we focus on sun spots occurrences and its associated properties.

The interest in finding features in solar images has increased dramatically ever since science was able to capture images of the sun. With the commission of the Solar Dynamics Observatory whose mission has been to observe the sun for finer features, make the data available for public, the interest in the research community has increased significantly. Various methods have been applied to find active regions on solar images; the methods vary from processing the whole image to selecting certain sections to modeling the region and then recognizing them on a large data set. The influence of solar activity is increased due to the increased communication flow in the upper atmosphere. The impact of solar activates on earth and spacecraft can be the evaluate using a measure called solar indices [4]. The magnetosphere and ionosphere has increased influence of solar activity at different latitudes [5]. One of the most widely used solar indices is the Wolf sunspot number that is based on the number of sunspots and sunspot groups. Sunspot number counts are taken at several solar observatories like Zurich Observatory (past), Ebro Observatory and SIDC, located in the Royal Observatory of Belgium they are used to calculate the relative Wolf sunspot number [6]. J J Curto developed the automatic 
identification of sunspots using mathematical al morphology tools based on images at Ebro observatory, it focused on the position and area of sunspots [7]. Improved version of sunspot detection having more geometric parameters were introduced by Zharkov, which consist of classification of sunspots groups like umbra and penumbra [8]. Further edge-detection methods are applied to find sunspot candidates followed by local thresholding using statistical properties of the region around sunspots by Zharkov, Zharkova and Ipson [9].

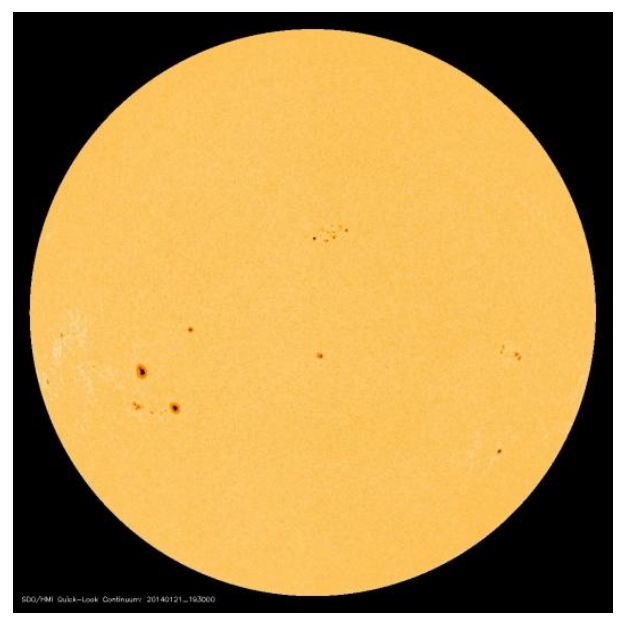

Fig 1. SDO/HMI Continuum (21-01-2014 19:30:00)

\section{IMAge PREPROCESSING}

The The identification of sunspots is done from Continuum and Magnetogram images provided by Helioseismic and Magnetic Imager (HMI) Instrument at the Solar Dynamics Observatory project. Michelson Doppler Imager (MDI) is the predecessor to the current Helioseismic and Magnetic Imager. The Solar Dynamics Observatory is the first mission to be launched for NASA's Living with a Star (LWS) Program, a program designed to understand the causes of solar variability and its impacts on Earth. SDO is designed to help us understand the Sun's influence on Earth and Near-Earth space by studying the solar atmosphere on small scales of space and time and in many wavelengths simultaneously. HMI will observe the full solar disk in the Fe I absorption line at $6173 \AA$ with a resolution of 1 arc-second. HMI consists of a refracting telescope, a polarization selector, an image stabilization system, a narrow band tunable filter and two 4096 pixel CCD cameras with mechanical shutters and control electronics. The continuous data rate is $55 \mathrm{Mbits} / \mathrm{s}$.

Images are made in a sequence of tuning and polarizations at a 4-second cadence for each camera. One camera is dedicated to a 45 s Doppler and line-of-sight field sequence while the other to a 90 s vector field sequence. The images acquired from HMI are preprocessed with median filter and is noise portion is filtered out. Compared to ground based images the space-borne images have low levels of noise but distortion may vary. The archived solar images are sometimes unsuitable because of their shape and variation of background intensity over the solar disk for the immediate application of segmentation algorithms (Walton et al. 1998; Bornmann et al. 1996). These may be due to several causes: the nature of the imaging instrument, slit position, inclination and the time taken to capture an image in particular spectral line; the nature of the solar atmosphere line-on-sight thickness that changes from disk centre to limb leading to the centre-to-limb darkening seen in visible emission (Irbah et al. 1999) or brightening seen in UV emission; the variations of transparency, turbulence etc. in the terrestrial atmospheric. Since feature detection techniques are to be applied automatically on a large number of images, all digital images are required to be pre-processed to a similar standard in order to satisfy the appropriate quality criteria. In the images acquired, the solar disk darkens towards the limb. To improve the accuracy of feature recognition near the limb, these variations are removed prior to detection using intensity standardization. We can apply median filter to reduce the variations in the intensity over image. The median filter eliminates the highest and lowest values to give a first approximation of the background fluctuations which can then be subtracted from the original image [10]. 


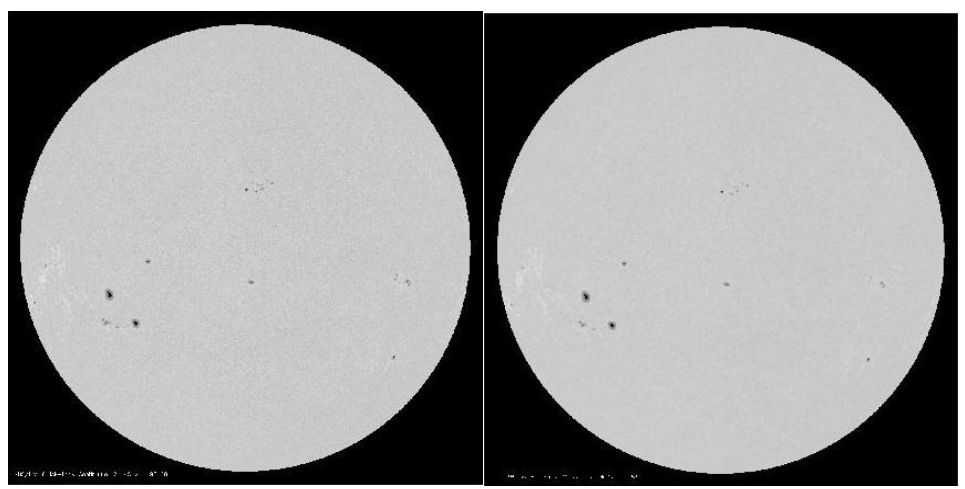

Fig 2. Grayscale Image and after applying median filtering of solar images

\section{DETECTION OF SUNSPOTS}

In the ideal case, the result of applying an edge detector to an solar image may lead to a set of connected curves that indicate the boundaries of objects, the boundaries of surface markings as well curves that correspond to discontinuities in surface orientation. Thus, applying an edge detector to an image may significantly reduce the amount of data to be processed and may therefore filter out information that may be regarded as less relevant, while preserving the important structural properties of an image. If the edge detection step is successful, the subsequent task of interpreting the information contents in the original image may therefore be substantially simplified. Unfortunately, however, it is not always possible to obtain such ideal edges from solar images of moderate complexity. Edges obtained from solar images often hampered by fragmentation, meaning that the edge curves are not connected, missing edge segments as well as false edges not corresponding to interesting phenomena in the image thus complicating the subsequent task of interpreting the image data.

The canny considered the mathematical problem of deriving an optimal smoothing filter given the criteria of detection, localization and minimizing multiple responses to a single edge [1]. He showed that the optimal filter given these assumptions is a sum of four exponential terms. He also showed that this filter can be well approximated by first-order derivatives of Gaussians. Canny also introduced the notion of non-maximum suppression, which means that given the pre-smoothing filters, edge points are defined as points where the gradient magnitude assumes a local maximum in the gradient direction. The technique of scale multiplication is analyzed in the framework of canny edge detection. A scale multiplication function is defined as the product of the responses of the detection filter at two scales. Edge maps are constructed as the local maxima by thresholding the scale multiplication results. The detection and localization criteria of the scale multiplication are derived. At a small loss in the detection criterion, the localization criterion can be much improved by scale multiplication [2]. The product of the two criteria for scale multiplication is greater than that for a single scale, which leads to better edge detection performance. This method is known as canny scale multiplication edge detection (CSM). Edges carry important information of an image. The common approach is to apply the first (or second) derivative to the smoothed image and then find the local maxima (or zero-crossings). Small-scaled filters are sensitive to edge signals but also prone to noise, whereas large-scaled filters are robust to noise but could filter out fine details. The multiple scales could be employed to describe and synthesize the varieties of edge structures [12]. The idea of scale multiplication was first exploited, where it is shown that the scale products can improve the edge localization and edge structures present observable magnitudes along the scales, while noise decreases rapidly. We show that through scale multiplication the localization accuracy can be significantly improved with only a small loss in the detection criterion and the product of the two criteria for the scale multiplication is greater than that for a single scale, leading to superior edge detection results. A simple but efficient edge detector by scale multiplication is then proposed. Ant Colony Optimization (ACO) is a population based met heuristic approach to find approximate solutions to difficult optimization problems. The inspiring source of ACO is the pheromone trail laying behavior of real ants, which use pheromone as a communication medium [3]. In analogy to the biological example, ACO is modeled based on the indirect communication of a colony of simple agents, called artificial ants, mediated by artificial pheromone trails. These pheromone trail values are modified at runtime based on a problem-dependent heuristic function and the amount of pheromone deposited by the ants while they traverse between their colony and a food source. The problem-dependent heuristic function, in the case of famous ACO algorithms for travelling salesman problem, is set to be the inverse of the distance between one city and another city [11]. In ACO, pheromone trail values serve as distributed, numerical information, which the ants use to construct solutions probabilistically. There is one solution per ant. The higher the pheromone value (initial edge), the higher the probability of an ant choosing that particular trail will be. The pheromone values on lower quality trails which are not reinforced often enough will 
progressively evaporate. The Pheromone based evaporation implements a useful form of forgetting: it avoids the algorithm from converging too rapidly toward a suboptimal region (final edge map), therefo es mentioned above are repeatedly applied until a termination condition is satisfied. In practice, a termination condition may be the maximum number of solutions generated, the maximum CPU time elapsed, or the maximum number of iterations without improvement in solution re favoring the exploration of new areas in the search space [4]. In the hybrid edge detection method (HM) the optimized canny filter output for different application is given an input for ant colony optimization. The edges in edge detected using canny scale multiplication are considered as the pheromone trail laying behavior of real ants, which use pheromone as a communication medium. The weak pheromone trail values are modified at runtime based on a problem-dependent heuristic function and the amount of pheromone deposited by the ants while they traverse between their colony and a food source. The weak pheromone trail values are due to the noise and other incorrect edge detected in the initial stage. The higher the pheromone value or initial edge strength, the higher the probability of an ant choosing that particular trail will be. The pheromone values on lower quality trails or weak edge which are not reinforced often enough will progressively evaporate [13].

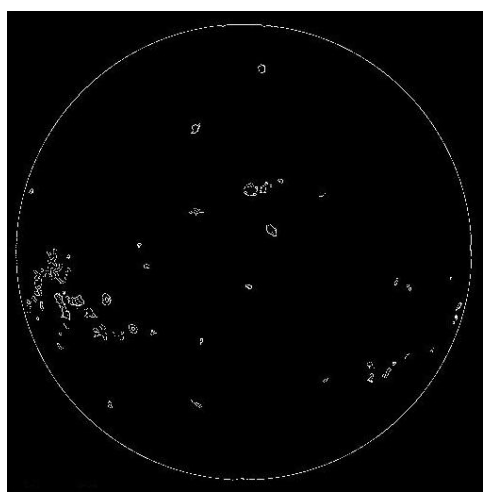

Fig 3. Sunspot Detected Image

\section{EXPERIMENTAL ANALYSIS}

We applied the sunspot detection and identification procedure to several images, obtained from the data archive of SOHO MDI Continuum Images. The Process for automatic detection of sunspots is done for the month of January 2014 and collected data from different images obtained. Comparison is done between manually detected and automatic detection. Solar Influences Data Center provides the sunspot numbers. $92 \%$ automatically detected sunspot match with of manually detected sunspots. The false detection rate of automatically detected sunspots is $7 \%$ and overall performance provides a better result for detection. Time taken by algorithm to complete the process is less one minute under standard environment.

\section{CONCLUSION}

Automatic detection of sunspot in solar images based on new improved edge detection method is implemented. This method can be used for automatic sunspot detection on full disk solar images obtained in the white light from MDI Instrument on SOHO satellite. Automated image cleaning procedures for elimination of limb darkening is done and using median filter used to reduce the effect of noise and to resist over detection. A new edge detection algorithm defines the regions of interest possibly containing sunspots. Efficient and automatic sunspots identification leads to the accurate and better identification of sunspots, their variation and impact on earth. The detection result for the selected images of January 2014 shows good outcome, with those produced at National Observatory for Astronomy and Astrophysics (NOAA). The evaluation of the performance of this method specific to solar application shows the measures in the paper match with the qualitative analysis. By visual inspection or solar data processing center data we can verify our results. Detection accuracy can be improved using different combination of neural network with edge detection techniques. More feature identification of sunspots and exact impact on earth climate in terms of climatic parametric values can be done as the further work.

\section{Acknowledgements}

The images for research are taken from SOHO/MDI consortium. SOHO is a project of international cooperation between ESA and NASA. Images are also taken from NASA/SDO and the AIA, EVE, and HMI science teams. 


\section{REFERENCES}

HTTP://CSEP10.PHYS.UTK.EDU/ASTR162/LECT/SUN/SUNSPOTS.HTML

[1] Automatic sunspots detection on full-disk solar images using mathematical morphology

[2] http://sidc.oma.be/

[3] Lanzerotti, L.J.: 2001, Space weather effects on communications. In: Daglis, I.A. (ed.) Space Storms and Space Weather Hazards, Kluwer, Dordrecht, 313 - 334.

[4] Hargreaves, J.K.: 1992, The Solar-Terrestrial Environment, University Press.

[5] Clette, F., Berghmans, D., Vanlommel, P., Van der Linden, R.A.M., Koeckelenbergh, A., Wauters, L.: 2007,From the Wolf number to the International Sunspot index: 25 years of SIDC. Adv. Space Res. 40, $919-928$.

[6] J. J. Curto, M. Blanca, E. Martínez Automatic Sunspots Detection on Full-Disk Solar Images using Mathematical Morphology, Solar Physics, August 2008, Volume 250, Issue 2, pp 411-429

[7] Zharkov, S., Zharkova, V., Ipson, S.: 2005, Statistical properties of sunspots in 1996 - 2004: I. Detection, North - South asymmetry and area distribution. Solar Phys. 228, $377-397$

[8] Zharkov, S., Zharkova, V., Ipson, S., Benkhalil, A.: 2005, Technique for automatic recognition of sunspots on full-disk solar images. EURASIP J. Appl. Signal Process. 15, 2573 - 2584.

[9] Aboudarham, J. and Fuller, N. (2004). Automatic versus manual detection of solar filaments, In Proceedings of the Eighth International Conference on Knowledge-Based Intelligent nformation \& Engineering Systems, University of Wellington, NZ, Springer Verlag, (in press)

[10] M. Qu, F. Y. Shih, J. Ju, and H. Wang, “Automatic solar filament detection using image processing techniques,” Solar Phys., vol. 228, no. 1-2, pp. 119-135, May 2005.

[11] Zharkova, V. V. Ipson, S. Benkhalil , A. and Zharkov, S. "Feature recognition in solar images", Artificial Intelligence Review, vol. 23, pp. 209-266 (2005)

[12] D.Murugan, et al, "Hybrid edge detection Using Canny and ant colony optimization", Communications in information science and management engineering, Aug 2013, Vol.3 Issue 8, PP 402-405.ISSN-2224-7785 by world academic publishing. 